\title{
Decay rate of a weakly dissipative viscoelastic plate equation with infinite memory
}

Received: 12 June 2020 / Accepted: 2 September 2020 / Published online: 24 September 2020

(C) The Author(s) 2020

\begin{abstract}
In this paper, a weakly dissipative viscoelastic plate equation with an infinite memory is considered. We show a general energy decay rate for a wide class of relaxation functions. To support our theoretical findings, some numerical illustrations are presented at the end. The numerical solution is computed using the popular finite element method in space, combined with time-stepping finite differences.
\end{abstract}

Mathematics Subject Classfication $35 \mathrm{~B} 40 \cdot 35 \mathrm{~L} 90 \cdot 45 \mathrm{~K} 05 \cdot 65 \mathrm{M} 06 \cdot 65 \mathrm{M} 60$

\section{Introduction}

The modeling of generalized Kirchhoff viscoelastic plate, where a bending moment relation with infinite memory is considered, can be described by the following weakly dissipative viscoelastic equation:

$$
\begin{cases}u^{\prime \prime}+\Delta^{2} u+\int_{0}^{\infty} g(s) \Delta u(t-s) \mathrm{d} s=0 & \text { in } \Omega \times(0, \infty), \\ u(x,-t)=u_{0}(x, t), \quad u^{\prime}(x, 0)=u_{1}(x), & \text { in } \Omega\end{cases}
$$

subject to the homogeneous conditions $u=\Delta u=0$ on $\partial \Omega$, where the physical domain $\Omega \subset \mathbb{R}^{n}$ is a bounded domain with a smooth (or piecewise smooth) boundary $\partial \Omega$. The history condition $u(x,-t)=u_{0}(x, t)$ means that we are taking into account all the deformation the material has undergone before the instant $t=0$. The initial velocity $u_{1}$ and the non-negative relaxation function $g$ are given.

The asymptotic or decaying behaviour, of different types of viscoelastic equations, including finite and infinite memories also linear and nonlinear dampings, were the subject of study of many researchers since the pioneer work of Dafermos [4,5]. The achieved energy decaying in the literature varies between (fractional) polynomial and exponential rates. This depends on the damping term, the memory term, the relaxation function, and the differential operator. In the presence of memory, the main common challenge was showing the energy decaying for most general relaxation function $g$, we refer the readers to $[1-3,6,7,9-11,13]$.

K. Anaya $(\bowtie) \cdot$ K. Mustapha

Department of Mathematics and Statistics, King Fahd University of Petroleum and Minerals,

Dhahran 31261, Kingdom of Saudi Arabia

E-mail: khalil.anaya1@gmail.com

K. Mustapha

E-mail: kassem@kfupm.edu.sa

S. A. Messaoudi

Department of Mathematics, University of Sharjah, 27272 Sharjah, UAE

E-mail: smessaoudi@sharjah.ac.ae 
Assuming that the relaxation function $g$ in (1.1) satisfies $g^{\prime}(t) \leq-\delta g(t)$, for $t \geq 0$ and for some positive constant $\delta$, Revira et al. [15] showed an exponential convergence of the energy decay. The main focus of this work was on investigating the energy decay of problem (1.1) but for a wider class of $g$, see (2.2) below. Owing to the presence of the weakly dissipative term, a second energy functional is introduced to achieve our goal. To make use of the convolution properties and the history condition, the viscoelastic (infinite memory) term is decomposed accordingly. For numerical illustrations of our theoretical finding, we provide a graphical illustration of the numerical energy decay using an approximate solution of problem (1.1) based on finite differences in time and finite elements in space.

Outline of the paper In the next section, we introduce some necessary notations and assumptions. For our decaying analysis, we state and prove a few technical lemmas. Section 3 is dedicated to show the decaying rates of the energy functional $E$, see Theorem 3.4. Having a weakly dissipative term in problem (1.1) leads us to introduce a second energy functional $\mathcal{E}$ [see (3.4)] to overcome the difficulties in proving the decay of $E$. For the sake of illustrating the theoretical decaying rate of $E$ numerically, we develop a fully discrete numerical method in Sect. 4. To avoid dealing with $\mathcal{C}^{2}$ numerical methods in the spatial variable (which is often not convenient on various physical domains $\Omega$ ) due to the presence of the biharmonic operator in problem (1.1), we rewrite (1.1) as a coupled system that involves a second-order elliptic operator instead. Then, we apply the $\mathcal{C}^{0}$ Galerkin finite element method to discretize in space. In the time variable, a second central difference is used to handle the second time derivative, while the other terms are approximated appropriately. We show the decaying of both, the numerical solution of problem (1.1) and also the approximation of the energy functional E.

\section{Preliminaries}

For $\ell \geq 0, H^{\ell}(\Omega)$ is the standard Sobolev space which reduces to $L^{2}(\Omega)$ space when $\ell=0$. On this space, $\langle\cdot, \cdot\rangle$ is the usual inner product and $\|\cdot\|$ is the associated $L^{2}(\Omega)$-norm.

An application of the Poincare inequality and using the elliptic regularity property,

$$
\frac{1}{\omega_{0}}\|\nabla w\|^{2} \leq\|\Delta w\|^{2} \leq \omega_{1}\|\nabla(\Delta w)\|^{2}, \forall w \in \mathcal{H}(\Omega),
$$

for some positive constants $\omega_{0}$ and $\omega_{1}$, where $\mathcal{H}(\Omega)=\left\{u \in H^{3}(\Omega): u=\Delta u=0\right.$ on $\left.\partial \Omega\right\}$.

In the decaying energy analysis (including Lemmas 2.1 and 2.2), we assume that the relaxation function $g \in \mathcal{C}^{1}\left(\mathbb{R}^{+}\right)$and satisfies

$$
g(0)>0, \quad 1-\max \left\{\omega_{0}, \omega_{1}\right\} \mu^{0}=: l>0, \quad \text { and }-c_{0} g(t)<g^{\prime}(t) \leq-\xi(t) g(t), \quad \forall t \geq 0,
$$

where $\xi$ is a positive non-increasing $\mathcal{C}^{1}$ function, $\mu^{0}=\int_{0}^{\infty} g(s) \mathrm{d} s$, and $c_{0}$ is a positive constant.

For later use, by (2.1) and the second inequality in (2.2), we have, for $t \geq 0$,

$$
\frac{1}{\mu^{0}}\|\nabla(\Delta u(t))\|^{2} \geq\|\Delta u(t)\|^{2} \geq\|\nabla u(t)\|^{2} \mu^{0} .
$$

For convenience, we introduce the following notations: for $t \geq 0$,

$$
(g \circ u)(t):=\int_{0}^{\infty} g(s)\left\|\eta^{t}(s)\right\|^{2} \mathrm{~d} s, \quad(g \circ[\nabla u, \Delta u])(t):=(g \circ \nabla u)(t)+(g \circ \Delta u)(t),
$$

and for $0<\varepsilon<1$,

$$
C_{\varepsilon}:=\int_{0}^{\infty} \frac{g^{2}(s)}{h_{\varepsilon}(s)} \mathrm{d} s \quad \text { with } \quad h_{\varepsilon}(t):=\varepsilon g(t)-g^{\prime}(t),
$$

where the function $\eta^{t}$ is the relative history of $u$ [5], defined as $\eta^{t}(s)=u(t)-u(t-s)$.

The next two lemmas will be used in the forthcoming decaying analysis section:

Lemma 2.1 [8] For any $v \in L_{l o c}^{2}\left([0,+\infty) ; L^{2}(\Omega)\right)$,

$$
\left\|\int_{0}^{\infty} g(s)(v(t-s)-v(t)) d s\right\|^{2} \leq C_{\varepsilon}\left(h_{\varepsilon} \circ v\right)(t), \quad \forall t \geq 0 .
$$


Lemma 2.2 [14] For any $\varphi \in H^{1}\left([0, \infty) ; L^{2}(\Omega)\right)$,

$$
\int_{0}^{\infty}\left\langle g(s) \varphi(t-s), \varphi^{\prime}(t)\right\rangle d s=\frac{1}{2} \frac{d}{d t}\left[\mu^{0}\|\varphi(t)\|^{2}-(g \circ \varphi)(t)\right]+\frac{1}{2}\left(g^{\prime} \circ \varphi\right)(t) .
$$

\section{Decay}

In this section, we find the energy functional $E$ of problem (1.1). As a starting point, taking the inner product of (1.1) with $u^{\prime}$, and then applying Green's formula (twice for the second term and once for the third term) and using the fact that $u^{\prime}=\Delta u^{\prime}=0$ on $\partial \Omega$, yield the following weak formulation of (1.1):

$$
\left\langle u^{\prime \prime}(t), u^{\prime}(t)\right\rangle+\left\langle\Delta u(t), \Delta u^{\prime}(t)\right\rangle-\int_{0}^{\infty} g(s)\left\langle\nabla u(t-s), \nabla u^{\prime}(t)\right\rangle \mathrm{d} s=0 .
$$

Using Lemma 2.2 with $\varphi=\nabla u$, this equation can be rewritten as follows:

$$
E^{\prime}(t)=\frac{1}{2}\left(g^{\prime} \circ \nabla u\right)(t) \leq 0 .
$$

where $E$ is the first energy functional given by

$$
E(t):=\frac{1}{2}\left[\left\|u^{\prime}(t)\right\|^{2}+\|\Delta u(t)\|^{2}-\mu^{0}\|\nabla u(t)\|^{2}+(g \circ \nabla u)(t)\right] \geq 0,
$$

where the non-negative property of $E$ follows from (2.3).

Now, taking the inner product of (1.1) with $-\Delta u^{\prime}$, following the above steps, we get

$$
\mathcal{E}^{\prime}(t)=\frac{1}{2}\left(g^{\prime} \circ \Delta u\right)(t) \leq 0,
$$

where $\mathcal{E}$ is the second energy functional, defined as follows:

$$
\mathcal{E}(t):=\frac{1}{2}\left[\left\|\nabla u^{\prime}(t)\right\|^{2}+\|\nabla(\Delta u(t))\|^{2}-\mu^{0}\|\Delta u(t)\|^{2}+(g \circ \Delta u)(t)\right] \geq 0,
$$

and its non-negativity follows also from (2.3). In the remaining analysis, $c$ is a generic positive constant. We estimate in the next two lemmas the time derivative of the following functionals:

$$
I_{1}(t)=\left\langle u^{\prime}(t), u(t)\right\rangle \text { and } I_{2}(t)=-\int_{0}^{\infty}\left\langle\eta^{t}(s), u^{\prime}(t)\right\rangle g(s) \mathrm{d} s .
$$

Lemma 3.1 Along the solution of (1.1), we have

$$
I_{1}^{\prime}(t) \leq\left\|u^{\prime}(t)\right\|^{2}-\frac{l}{2}\|\Delta u(t)\|^{2}+c C_{\varepsilon}\left(h_{\varepsilon} \circ \nabla u\right)(t) .
$$

Proof Since $I_{1}^{\prime}(t)=\left\|u^{\prime}(t)\right\|^{2}+\left\langle u^{\prime \prime}(t), u(t)\right\rangle$, using (1.1) and Green's formula, we get

$$
I_{1}^{\prime}(t)=\left\|u^{\prime}(t)\right\|^{2}-\|\Delta u(t)\|^{2}+\mu^{0}\|\nabla u(t)\|^{2}-\int_{0}^{\infty} g(s)\left\langle\nabla \eta^{t}(s), u(t)\right\rangle \mathrm{d} s .
$$

Young's inequality, Lemma 2.1, and the inequalities in (2.1) imply that

$$
\begin{aligned}
\mu^{0}\|\nabla u(t)\|^{2}-\int_{0}^{\infty} g(s)\left\langle\nabla \eta^{t}(s), u(t)\right\rangle \mathrm{d} s & \leq \omega_{0} \mu^{0}\|\Delta u(t)\|^{2}+\frac{l}{2 \omega_{0}}\|\nabla u(t)\|^{2}+\frac{\omega_{0}}{2 l}\left\|\int_{0}^{\infty} \nabla \eta^{t}(s) g(s) \mathrm{d} s\right\|^{2} \\
& \leq(1-l)\|\Delta u(t)\|^{2}+\frac{l}{2}\|\Delta u(t)\|^{2}+c C_{\varepsilon}\left(h_{\varepsilon} \circ \nabla u\right)(t) .
\end{aligned}
$$

Combining the above results completes the proof. 
Lemma 3.2 Along the solution of (1.1) and for $\delta>0$, we have

$$
I_{2}^{\prime}(t) \leq \delta\|\Delta u(t)\|^{2}-\left(\mu^{0}-\delta\right)\left\|u^{\prime}(t)\right\|^{2}+\frac{c}{\delta}\left(C_{\varepsilon}+1\right)\left(h_{\varepsilon} \circ \Delta u\right)(t) .
$$

Proof Differentiating $I_{2}$ and using the differential equation in (1.1), we get

$$
I_{2}^{\prime}(t)=I_{2,1}(t)+I_{2,2}(t)+I_{2,3}(t)-\mu^{0}\left\|u^{\prime}(t)\right\|^{2}
$$

where

$$
\begin{aligned}
& I_{2,1}(t)=\int_{0}^{\infty} g(s)\left\langle\eta^{t}(s), \Delta^{2} u(t)\right\rangle \mathrm{d} s, \quad I_{2,2}(t)=\left\langle\int_{0}^{\infty} g(s) \Delta u(t-s) \mathrm{d} s, \int_{0}^{\infty} g(s) \eta^{t}(s) \mathrm{d} s\right\rangle \\
& I_{2,3}(t)=\left\langle\int_{0}^{\infty} g(s) \eta_{s}^{t}(s) \mathrm{d} s, u^{\prime}(t)\right\rangle .
\end{aligned}
$$

By Green's formula, Young's inequality and Lemma 2.1, we have

$$
I_{2,1}(t) \leq\|\Delta u(t)\| \int_{0}^{\infty} g(s)\left\|\Delta \eta^{t}(s)\right\| \mathrm{d} s \leq \frac{\delta}{2}\|\Delta u(t)\|^{2}+\frac{c}{\delta} C_{\varepsilon}\left(h_{\varepsilon} \circ \Delta u\right)(t),
$$

and in addition, using $u(t-s)=u(t)-\eta^{t}(s)$,

$$
\begin{aligned}
I_{2,2}(t) & =\left\|\int_{0}^{\infty} g(s) \nabla \eta^{t}(s) \mathrm{d} s\right\|^{2}-\left\langle\int_{0}^{\infty} g(s) \nabla u(t) \mathrm{d} s, \int_{0}^{\infty} g(s) \nabla \eta^{t}(s) \mathrm{d} s\right\rangle \\
& \leq C_{\varepsilon}\left(h_{\varepsilon} \circ \nabla u\right)(t)+c\|\nabla u(t)\| \int_{0}^{\infty} g(s)\left\|\nabla \eta^{t}(s)\right\| \mathrm{d} s \\
& \leq C_{\varepsilon}\left(h_{\varepsilon} \circ \nabla u\right)(t)+\frac{\delta}{2 \omega_{0}}\|\nabla u(t)\|^{2}+\frac{c}{\delta} C_{\varepsilon}\left(h_{\varepsilon} \circ \nabla u\right)(t) .
\end{aligned}
$$

To estimate $I_{2,3}(t)$, we perform integration by parts and then make use of Young's and Hölder's inequalities, the fact that $g^{\prime}=\varepsilon g-h_{\varepsilon}$ and Lemma 2.1. So, we obtain

$$
\begin{aligned}
I_{2,3}(t) & =-\left\langle\int_{0}^{\infty} g^{\prime}(s) \eta^{t}(s) \mathrm{d} s, u^{\prime}(t)\right\rangle \\
& =-\varepsilon\left\langle\int_{0}^{\infty} g(s) \eta^{t}(s) \mathrm{d} s, u^{\prime}(t)\right\rangle+\left\langle\int_{0}^{\infty} h_{\varepsilon}(s) \eta^{t}(s) \mathrm{d} s, u^{\prime}(t)\right\rangle \\
& \leq \frac{\delta}{2}\left\|u^{\prime}(t)\right\|^{2}+\frac{\varepsilon^{2}}{2 \delta}\left\|\int_{0}^{\infty} g(s) \eta^{t}(s) \mathrm{d} s\right\|^{2}+\frac{\delta}{2}\left\|u^{\prime}(t)\right\|^{2}+\frac{1}{2 \delta}\left\|\int_{0}^{\infty} h_{\varepsilon}(s) \eta^{t}(s) \mathrm{d} s\right\|^{2} \\
& \leq \delta\left\|u^{\prime}(t)\right\|^{2}+\frac{c}{\delta}\left(h_{\varepsilon} \circ u\right)(t)+\frac{1}{2 \delta}\left(\int_{0}^{\infty} h_{\varepsilon}^{\frac{1}{2}}(s) h_{\varepsilon}^{\frac{1}{2}}(s)\left\|\eta^{t}(s)\right\| \mathrm{d} s\right)^{2} .
\end{aligned}
$$

Inserting the obtained estimates of $I_{2,1}(t), I_{2,2}(t)$, and $I_{2,3}(t)$ in (3.7) and using

$$
\left(\int_{0}^{\infty} h_{\varepsilon}^{\frac{1}{2}}(s) h_{\varepsilon}^{\frac{1}{2}}(s)\left\|\eta^{t}(s)\right\| \mathrm{d} s\right)^{2} \leq \int_{0}^{\infty} h_{\varepsilon}(s) \mathrm{d} s \int_{0}^{\infty} h_{\varepsilon}(s)\left\|\eta^{t}(s)\right\|^{2} \mathrm{~d} s \leq c\left(h_{\varepsilon} \circ u\right)(t)
$$

(by Young's and Hölder's inequalities), in addition to the elliptic regularity property of the operator $-\Delta$, the desired bound followed.

Lemma 3.3 For $\varepsilon_{1}, \varepsilon_{2}>0$, the functional $\mathscr{L}(t):=N(E(t)+\mathcal{E}(t))+\varepsilon_{1} I_{1}(t)+\varepsilon_{2} I_{2}(t)$ satisfies $\mathscr{L} \sim E+\mathcal{E}$ for a sufficiently large $N$ (the proof is in [12]). Moreover, there exist positive constants $\alpha_{1}$ and $\alpha_{1}$, such that

$$
\mathscr{L}^{\prime}(t) \leq-\alpha_{1} E(t)+\alpha_{2} \int_{0}^{\infty} g(s)\left(\left\|\nabla \eta^{t}(s)\right\|^{2}+\left\|\Delta \eta^{t}(s)\right\|^{2}\right) d s, \quad \forall t \geq 0 .
$$


Proof To prove the estimate in (3.8), we differentiate $\mathscr{L}$ and use (3.1) and (3.3), in addition to Lemmas 3.1 and 3.2 . Hence,

$$
\begin{aligned}
& \mathscr{L}^{\prime}(t) \leq \frac{N}{2}\left(g^{\prime} \circ[\nabla u, \Delta u]\right)(t)+\varepsilon_{1}\left[\left\|u^{\prime}(t)\right\|^{2}-\frac{l}{2}\|\Delta u(t)\|^{2}+c C_{\varepsilon}\left(h_{\varepsilon} \circ \nabla u\right)(t)\right] \\
& \quad+\varepsilon_{2}\left[\delta\|\Delta u(t)\|^{2}-\left(\mu^{0}-\delta\right)\left\|u^{\prime}(t)\right\|^{2}+\frac{c}{\delta}\left(C_{\varepsilon}+1\right)\left(h_{\varepsilon} \circ \Delta u\right)(t)\right] .
\end{aligned}
$$

Recalling that $g^{\prime}(t)=\varepsilon g(t)-h_{\varepsilon}(t)$, then rearranging the terms in the above equation, and noting that $h_{\varepsilon}>0$, we arrive at

$$
\begin{aligned}
& \mathscr{L}^{\prime}(t) \leq-\left[\left(\mu^{0}-\delta\right) \varepsilon_{2}-\varepsilon_{1}\right]\left\|u^{\prime}\right\|^{2}-\left(\frac{l}{2} \varepsilon_{1}-\varepsilon_{2} \delta\right)\|\Delta u\|^{2}+\frac{N \varepsilon}{2}(g \circ[\nabla u, \Delta u]) \\
& +\left[\frac{c}{\delta}\left(1+C_{\varepsilon}\right)\left(\varepsilon_{1}+\varepsilon_{2}\right)-\frac{N}{2}\right]\left(h_{\varepsilon} \circ \Delta u\right)(t) .
\end{aligned}
$$

Choosing $\varepsilon=\frac{1}{N}$. Since $\frac{g^{2}(s)}{\varepsilon g(s)-g^{\prime}(s)} \leq \frac{g(s)}{\varepsilon}, C_{\varepsilon} \leq N \mu^{0}$. Hence, for $N \geq 2 \frac{c}{\delta}\left(1+N \mu^{0}\right)\left(\varepsilon_{1}+\varepsilon_{2}\right)$ (which is valid for $\varepsilon_{1}$ and $\varepsilon_{2}$ are sufficiently small), we notice from the above equation that

$$
\mathscr{L}^{\prime}(t) \leq-\left[\left(\mu^{0}-\delta\right) \varepsilon_{2}-\varepsilon_{1}\right]\left\|u^{\prime}\right\|^{2}-\left(\frac{l}{2} \varepsilon_{1}-\varepsilon_{2} \delta\right)\|\Delta u\|^{2}+\frac{1}{2}(g \circ[\nabla u, \Delta u]) .
$$

Choosing $\delta<\frac{l}{8} \mu^{0}<\frac{1}{8} \mu^{0}$ (because $0<l<1$ ) and $\varepsilon_{1}=\frac{3}{8} \mu^{0} \varepsilon_{2}$, after some calculations, we have

$$
\left(\mu^{0}-\delta\right) \varepsilon_{2}-\varepsilon_{1}>\frac{1}{2} \mu^{0} \varepsilon_{2}, \frac{l}{2} \varepsilon_{1}-\delta \varepsilon_{2}>\frac{l}{16} \mu^{0} \varepsilon_{2}
$$

and consequently,

$$
\mathscr{L}^{\prime}(t) \leq-\frac{1}{2} \mu^{0} \varepsilon_{2}\left\|u^{\prime}\right\|^{2}-\frac{l}{16} \mu^{0} \varepsilon_{2}\|\Delta u\|^{2}+\frac{1}{2}(g \circ[\nabla u, \Delta u])(t) .
$$

Finally, recalling the definition of $E$ and $\eta^{t}$, and using the above bound, we obtain the desired estimate.

We are ready now to estimate the energy functional associated with the problem (1.1).

Theorem 3.4 Assume that $u_{0}(., 0) \in \mathcal{H}(\Omega)$ with $\max \left\{\left\|\nabla u_{0}(., s)\right\|,\left\|\Delta u_{0}(., s)\right\|\right\} \leq m$ (for some positive constant $m$ ), and $u_{1} \in H^{2}(\Omega) \cap H_{0}^{1}(\Omega)$. Then, for t large enough, there exists a positive constant $\delta>0$ such that

$$
E(t) \leq \delta\left(\frac{1+\int_{0}^{t} \xi(s) \int_{s}^{\infty} g(s) d s d s}{1+\int_{0}^{t} \xi(s) d s}\right) .
$$

Proof From the non-increasing property of $\xi$, the inequality $g^{\prime} \leq-\xi g$, we have for $t \geq 0$;

$$
\xi(t) \int_{0}^{t} g(s)\left\|\nabla \eta^{t}(s)\right\|^{2} \mathrm{~d} s \leq \int_{0}^{t}\left\|\nabla \eta^{t}(s)\right\|^{2} \xi(s) g(s) \mathrm{d} s \leq-\int_{0}^{t}\left\|\nabla \eta^{t}(s)\right\|^{2} g^{\prime}(s) \mathrm{d} s \leq-c_{0} E^{\prime}(t),
$$

for some positive constant $c_{0}$. However, owing to the inequality, $\|\nabla u(t)\|^{2} \leq \frac{2 \omega_{0}}{l} E(t) \leq \frac{2 \omega_{0}}{l} E(0)$, we have for $s>t$,

$$
\left\|\nabla \eta^{t}(s)\right\|^{2} \leq 2\|\nabla u(t)\|^{2}+2\|\nabla u(t-s)\|^{2} \leq \frac{4 \omega_{0}}{l} E(0)+2 \sup _{\zeta>0}\left\|\nabla u_{0}(\zeta)\right\|^{2} \leq \frac{4 \omega_{0}}{l} E(0)+2 m^{2},
$$

Consequently, we get

$$
\xi(t) \int_{t}^{\infty} g(s)\left\|\nabla \eta^{t}(s)\right\|^{2} \mathrm{~d} s \leq \xi(t)\left(\frac{4 \omega_{0}}{l} E(0)+2 m^{2}\right) \int_{t}^{\infty} g(s) \mathrm{d} s .
$$


Therefore, for $t \geq 0$,

$$
\xi(t) \int_{0}^{\infty} g(s)\left\|\nabla \eta^{t}(s)\right\|^{2} \mathrm{~d} s \leq-c_{0} E^{\prime}(t)+\xi(t)\left(\frac{4 \omega_{0}}{l} E(0)+2 m^{2}\right) \int_{t}^{\infty} g(s) \mathrm{d} s .
$$

Following similar arguments, and using the inequality $\|\Delta u(t)\|^{2} \leq \frac{2}{l} E(t) \leq \frac{2}{l} E(0)$, we obtain

$$
\left.\xi(t) \int_{0}^{\infty} g(s)\left\|\Delta \eta^{t}(s)\right\|^{2} \mathrm{~d} s \leq-c_{0} \mathcal{E}^{\prime}(t)\right)+\xi(t)\left(\frac{4}{l} E(0)+2 m^{2}\right) \int_{t}^{\infty} g(s) \mathrm{d} s .
$$

Multiplying (3.8) by $\xi(t)$, and using (3.9) and (3.10), we get

$$
\xi(t) \mathscr{L}^{\prime}(t) \leq-\alpha_{1} \xi(t) E(t)-\beta_{1}\left(E^{\prime}(t)+\mathcal{E}^{\prime}(t)\right)+\beta_{2} \xi(t) \int_{t}^{\infty} g(s) \mathrm{d} s,
$$

where $\beta_{1}=c_{0} \alpha_{2}$ and $\beta_{2}=\alpha_{2}\left(\frac{4}{l}\left(1+\omega_{0}\right) E(0)+2 m^{2}\right)$. Therefore, differentiating the functional $\mathscr{L}(t):=$ $\xi \mathscr{L}(t)+\beta_{1}(E(t)+\mathcal{E}(t))$ and using (3.11), we have

$$
\mathscr{L}^{\prime}(t) \leq \xi(t) \mathscr{L}^{\prime}(t)+\beta_{1}\left(E^{\prime}(t)+\mathcal{E}^{\prime}(t)\right) \leq-\alpha_{1} \xi(t) E(t)+\beta_{2} \xi(t) \int_{t}^{\infty} g(s) \mathrm{d} s .
$$

Integrating the above equation over $(0, t)$ yields

$$
\mathscr{L}(t) \leq \mathscr{L}(0)+\beta_{2} \int_{0}^{t} \xi(s) \int_{s}^{\infty} g(s) \mathrm{d} s \mathrm{~d} s-\alpha_{1} \int_{0}^{t} \xi(s) E(s) \mathrm{d} s .
$$

However, $E(s) \geq E(t)$ for $s \leq t$ (by the non-increasing property of $E$ ), and $\beta_{1} E(t) \leq \mathscr{L}(t)$ (from the definition of $\mathscr{L})$, so

$$
\left(\beta_{1}+\alpha_{1} \int_{0}^{t} \xi(s) \mathrm{d} s\right) E(t) \leq \mathscr{L}(0)+\beta_{2} \int_{0}^{t} \xi(s) \int_{s}^{\infty} g(s) \mathrm{d} s \mathrm{~d} s .
$$

Choosing $\delta=\frac{\max \left\{\mathscr{L}(0), \beta_{2}\right\}}{\min \left\{\beta_{1}, \alpha_{1}\right\}}$, then the desired energy bound is obtained.

\section{Numerical study}

This section is devoted to illustrate numerically the achieved theoretical decaying results in Theorem 3.4 on a two-dimensional test problem of the form (1.1) with space variables $x$ and $y$. We develop a numerical scheme for problem (1.1) using finite differences for the time discretization combined with the continuous Galerkin finite element method in space. Applying Galerkin method to problem (1.1) directly forces us to deal with $\mathcal{C}^{2}$ polynomial approximations, which is definitely not convenient owing to the complexity in constructing the basis functions on various physical domains, and also increase the cost of computations. To avoid this, we rewrite (1.1) as a coupled system of lower order elliptic problems:

$$
\begin{cases}u^{\prime \prime}-\Delta w+\int_{0}^{t} g(t-s) \Delta u(s) \mathrm{d} s=F & \text { in } \Omega \times(0, \infty), \\ w+\Delta u=0 & \text { in } \Omega \times(0, \infty), \\ u(x, y, 0)=u_{0}(x, y, 0), \quad u^{\prime}(x, y, 0)=u_{1}(x, y), & \text { in } \Omega\end{cases}
$$

with $u=w=0$ on $\partial \Omega$, where $w=-\Delta u$, and $F(x, y, t)=-\int_{t}^{\infty} g(s) \Delta u_{0}(x, y, s-t) \mathrm{d} s$.

Our numerical discretization in space is applicable to any bounded polygonal physical domain $\Omega$. However, and for simplicity, we choose $\Omega$ to be the unit square $(0,1) \times(0,1)$. Let $\mathcal{T}_{h}$ be a family of uniform $M^{2}$-square mesh-cells of $\Omega$ with diagonal $h=\sqrt{2} / M$ each. Let $V_{h} \subset H_{0}^{1}(\Omega)$ denote the usual space of continuous, piecewise-linear polynomials on $\mathcal{T}_{h}$ that vanish on $\partial \Omega$. To discretize in time, we truncate the interval $(0, \infty)$ 

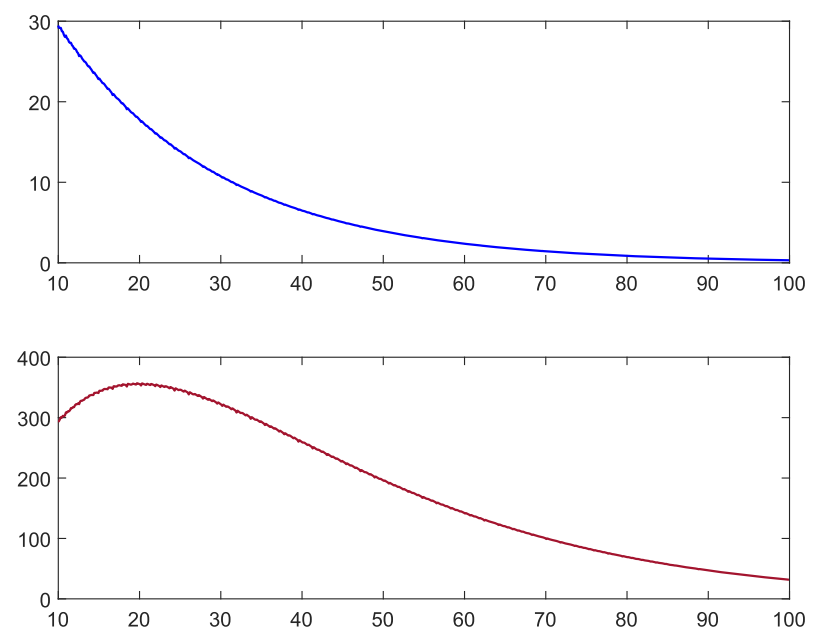

Fig. 1 The graphical plots of the numerical approximations of the energy $E(t)$ (top) and the weighted energy $t E(t)$ (bottom) the against $t$

and work instead on the finite interval $(0, T]$ with $T$ that can be sufficiently large. Divide $[0, T]$ uniformly into $N$ subintervals each with size $\tau:=T / N$ and nodes $\left\{t_{n}\right\}_{n=0}^{N}$. For a given grid function $v^{n}$, let

$$
\delta_{t t} v^{n}=\frac{v^{n+1}-2 v^{n}+v^{n-1}}{\tau^{2}}, \quad v^{n+\frac{1}{2}}=\frac{v^{n}+v^{n-1}}{2}, \quad v^{n+\frac{1}{4}}=\frac{v^{n+1}+2 v^{n}+v^{n-1}}{4} .
$$

Taking the inner product of the first two equations in (4.1) with $\phi, \psi \in H_{0}^{1}(\Omega)$, respectively, then, applying Green's formula and using $u=w=0$ on $\partial \Omega$. This implies

$$
\begin{cases}\left\langle u^{\prime \prime}, \phi\right\rangle+\langle\nabla w, \nabla \phi\rangle-\int_{0}^{t} g(t-s)\langle\nabla u(s), \nabla \phi\rangle \mathrm{d} s=\langle F, \phi\rangle, & \forall \phi \in H_{0}^{1}(\Omega), \\ \langle w, \psi\rangle-\langle\nabla u, \nabla \psi\rangle=0, & \forall \psi \in H_{0}^{1}(\Omega) .\end{cases}
$$

Motivated by the above formulation, our fully discrete numerical scheme is defined as follows: Find $U_{h}^{n+1}, W_{h}^{n+1} \in V_{h}$ such that

$$
\left\{\begin{array}{l}
\left\langle\delta_{t t} U_{h}^{n}, \phi_{h}\right\rangle+\left\langle\nabla W_{h}^{n+\frac{1}{4}}, \nabla \phi_{h}\right\rangle-\int_{0}^{t_{n+1}} g\left(t_{n+1}-s\right)\left\langle\nabla \bar{U}_{h}(s), \nabla \phi_{h}\right\rangle \mathrm{d} s=\left\langle F^{n+1}, \phi_{h}\right\rangle, \\
\left\langle W_{h}^{n+1}, \psi_{h}\right\rangle-\left\langle\nabla U_{h}^{n+1}, \nabla \psi_{h}\right\rangle=0
\end{array}\right.
$$

$\forall \phi_{h}, \psi_{h} \in V_{h}$, with $1 \leq n \leq N-1$, where the piecewise constant function (in time variable) $\bar{U}_{h}(s)=U_{h}^{j+\frac{1}{2}}$ for $t_{j}<s<t_{j+1}$ with $0 \leq j \leq N$, and $F^{n+1}=F\left(t_{n+1}\right)$.

Let $\left\{\phi_{p}\right\}_{p=1}^{d_{h}}$ be the two-dimensional hat basis functions of $V_{h}$. Then, $U_{h}^{n}$ and $W_{h}^{n}$ can be written in term of the basis functions as follows: $U_{h}^{n}=\sum_{i=1}^{d_{h}} b_{i}^{n} \phi_{p_{i}}$ and $W_{h}^{n}=\sum_{i=1}^{d_{h}} c_{i}^{n} \phi_{p_{i}}$. We define $d_{h} \times d_{h}$ matrices: $\boldsymbol{A}=\left[\left\langle\nabla \phi_{q}, \nabla \phi_{p}\right\rangle\right]$ and $\boldsymbol{M}=\left[\left\langle\phi_{q}, \phi_{p}\right\rangle\right]$. The $d_{h}$-dimensional constant column vectors $\mathbf{b}^{n}, \mathbf{c}^{n}$, and $\boldsymbol{F}^{n+1}$ are, respectively, the transpose of the vectors $\left[b_{1}^{n}, b_{2}^{n}, \ldots, b_{d_{h}}^{n}\right],\left[c_{1}^{n}, c_{2}^{n}, \ldots, c_{d_{h}}^{n}\right]$ and $\left[\left\langle F^{n+1}, \phi_{1}\right\rangle,\left\langle F^{n+1}, \phi_{2}\right\rangle, \ldots,\left\langle F^{n+1}, \phi_{d_{h}}\right\rangle\right]$.

Therefore, the fully discrete scheme (4.2) has the following matrix representation:

$$
\boldsymbol{M} \delta_{t t} \mathbf{b}^{n}+\boldsymbol{A} \mathbf{c}^{n+\frac{1}{4}}-g_{n+1, n} \boldsymbol{A} \mathbf{b}^{n+\frac{1}{2}}=\sum_{j=0}^{n-1} g_{n+1, j} \boldsymbol{A} \mathbf{b}^{j+\frac{1}{2}}+\boldsymbol{F}^{n+1}, \quad \boldsymbol{M} \mathbf{c}^{n+1}-\boldsymbol{A} \mathbf{b}^{n+1}=\mathbf{0},
$$

with $g_{n+1, j}:=\int_{t_{j}}^{t_{j+1}} g\left(t_{n+1}-s\right) \mathrm{d} s$. From the second equation, we notice that $\boldsymbol{A} \mathbf{c}^{n+1}=\boldsymbol{B} \mathbf{b}^{n+1}$ where $\boldsymbol{B}=\boldsymbol{A} \boldsymbol{M}^{-1} \boldsymbol{A}$. Substitute this in the first equation and rearranging the terms, 

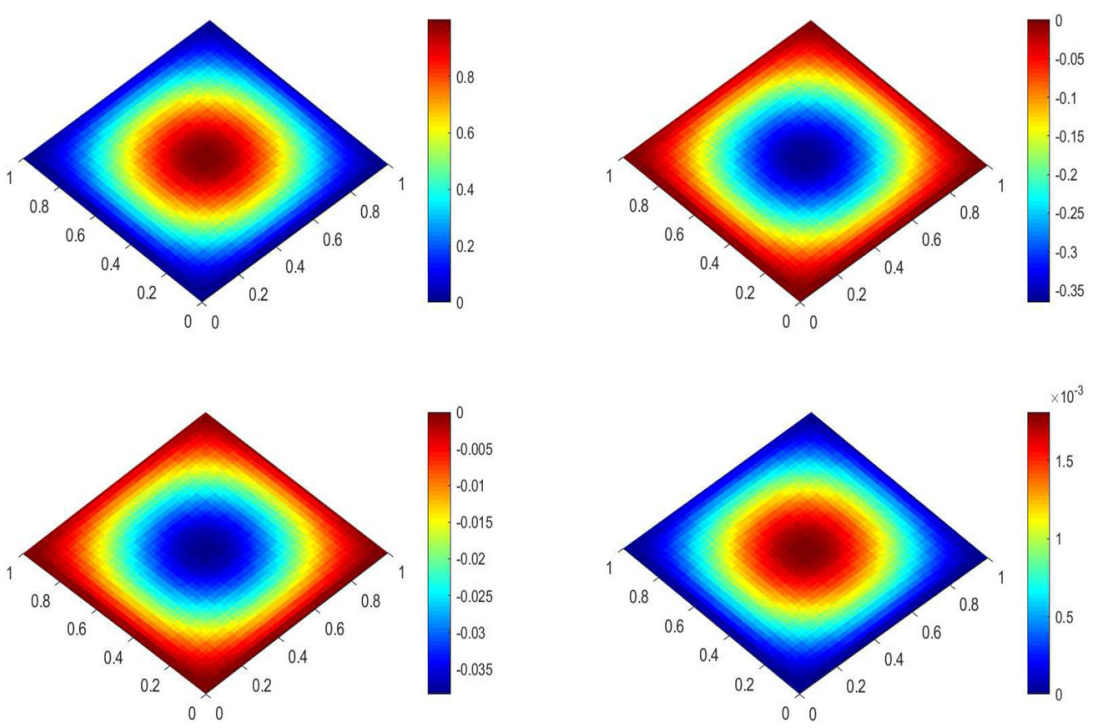

Fig. 2 The numerical solution plots for $t=0$ (top-left), $t=10$ (top-right), $t=50$ (bottom-left), and $t=100$ (bottom-right)

$$
\begin{aligned}
& {\left[4 \boldsymbol{M}+\tau^{2} \boldsymbol{B}-2 \tau^{2} g_{n+1, n} \boldsymbol{A}\right] \mathbf{b}^{n+1}=\boldsymbol{M}\left(8 \mathbf{b}^{n}-4 \mathbf{b}^{n-1}\right)-\tau^{2} \boldsymbol{B}\left(2 \mathbf{b}^{n}+\mathbf{b}^{n-1}\right)} \\
& +2 \tau^{2} g_{n+1, n} \boldsymbol{A} \mathbf{b}^{n}+2 \tau^{2} \sum_{j=0}^{n-1} g_{n+1, j} \boldsymbol{A}\left[\mathbf{b}^{j+1}+\mathbf{b}^{j}\right]+\boldsymbol{F}^{n+1}
\end{aligned}
$$

Therefore, at each time level $t_{n+1}$, the numerical coupled system (4.2) reduces to a finite square linear system, where the unknown is the column vector $\mathbf{b}^{n+1}$. Whence the coefficient vector $\mathbf{b}^{n+1}$ is computed, $\mathbf{c}^{n+1}$ can be determined by solving $\boldsymbol{M} \mathbf{c}^{n+1}=\boldsymbol{A} \mathbf{b}^{n+1}$, that is, $\mathbf{c}^{n+1}=\boldsymbol{M}^{-1} \boldsymbol{A} \mathbf{b}^{n+1}$. This will be used to compute the numerical energy function $E_{h}\left(t_{n}\right)$.

To solve linear system in (4.3) for $\mathbf{b}^{n+1}$, successively, the column vectors $\mathbf{b}^{0}$ and $\mathbf{b}^{1}$ need to be determined first. In other words, we have to compute the approximate solutions $U_{h}^{0}$ and $U_{h}^{1}$ first. We consider $U_{h}^{0}$ to be the Ritz projection of $u_{0}$ on the finite dimensional space $V_{h}$. However, motivated by the Taylor series expansion of $u$ in time about $t=0$, we choose $U_{h}^{1}$ to be the Ritz projection $u_{0}+t_{1} u_{1}$ on $V_{h}$. For sake of computing efficiently the spatial integrals in the linear system in (4.3), on each cell of our two-dimensional partition, the associated integral is approximated using 4-point (that is, 2-point in each direction) Gauss cubature rule.

In our numerical example, choose $T=150, u_{0}(x, y, t)=t^{2} \sin (\pi x) \sin (\pi y)$ and $u_{1}(x, y)=0$. We run our computer program with $M=20$ (that is, 400 cells in space) and $N=120000$. We choose $g(t)=e^{-t}$; then $g^{\prime}(t)=-\xi(t) g(t)$ with $\xi(t)=1$. Thus, by Theorem 3.4, we expect our energy to decay monomially, that is, $t E(t) \leq c$ for a sufficiently large $t$. This is confirmed in Fig. 1. In addition, the plot of the numerical solution $U_{h}$ in Fig. 2 shows its convergence to zero as the time $t$ getting far away from 0.

Acknowledgements The support of King Fahd University of Petroleum \& Minerals and University of Sharjah is gratefully acknowledged. The second author is sponsored by the University of Sharjah, Research group MASEP.

Open Access This article is licensed under a Creative Commons Attribution 4.0 International License, which permits use, sharing, adaptation, distribution and reproduction in any medium or format, as long as you give appropriate credit to the original author(s) and the source, provide a link to the Creative Commons licence, and indicate if changes were made. The images or other third party material in this article are included in the article's Creative Commons licence, unless indicated otherwise in a credit line to the material. If material is not included in the article's Creative Commons licence and your intended use is not permitted by statutory regulation or exceeds the permitted use, you will need to obtain permission directly from the copyright holder. To view a copy of this licence, visit http://creativecommons.org/licenses/by/4.0/.

\section{References}

1. Al-Gharabli, M.; Guesmia, A.; Messaoudi, S.: Existence and a general decay results for a viscoelastic plate equation with a logarithmic nonlinearity. Commun. Pure Appl. Anal. 18, 159-175 (2019) 
2. Belhannache, F.; Al-Gharabli, M.; Messaoudi, S.: Asymptotic stability for a viscoelastic equation with nonlinear damping and very general type of relaxation functions. J. Dyn. Control Syst. 26, 45-67 (2019)

3. Cavalcanti, M.; Cavalcanti, V.; Soriano, J.: Exponential decay for the solution of semilinear viscoelastic wave equations with localized damping. Electron. J. Differ. Equ. 44, 54-65 (2002)

4. Dafermos, M.: An abstract Volterra equation with applications to linear viscoelasticity. J. Differ. Equ. 7, 554-569 (1970)

5. Dafermos, M.: Asymptotic stability in viscoelasticity. Arch. Ration. Mech. Anal. 37, 297-308 (1970)

6. Guesmia, A.: Asymptotic stability of abstract dissipative systems with infinite memory. J. Math. Anal. Appl. 382, 748-760 (2011)

7. Hassan, J.; Messaoudi, S.: General decay rate for a class of weakly dissipative second-order systems with memory. Math. Methods Appl. Sci. 42, 2842-2853 (2019)

8. Jin, K.; Liang, J.; Xiao, T.: Coupled second order evolution equations with fading memory: optimal energy decay rate. J. Differ. Equ. 257, 1501-1528 (2014)

9. Komornik, V.: On the nonlinear boundary stabilization of Kirchhoff plates. Nonlinear Differ. Equ. Appl. 1(4), 323-337 (1994)

10. Lagnese, J.: Asymptotic energy estimates for Kirchhoff plates subject to weak viscoelastic damping. Int. Ser. Numer. Math. 91, 211-236 (1989)

11. Liu, W.: General decay rate estimate for a viscoelastic equation with weakly nonlinear time-dependent dissipation and source terms. J. Math. Phys. 50(11), 113-130 (2009)

12. Messaoudi, S.; Al-Gharabli, M.: A general stability result for a nonlinear wave equation with infinite memory. Appl. Math. Lett. 26(11), 1082-1086 (2013)

13. Rivera, J.; Eugenio, C.; Rioco, B.: Decay rates for viscoelastic plates with memory. J. Elast. 44, 61-87 (1996)

14. Rivera, J.; Reinhard, R.: Magneto-thermo-elasticity-large-time behavior for linear systems. Adv. Differ. Equ. 6(3), 359-384 (2001)

15. Rivera, J.; Maria, N.: Optimal energy decay rate for a class of weakly dissipative second-order systems with memory. Appl. Math. Lett. 23, 743-746 (2010)

Publisher's Note Springer Nature remains neutral with regard to jurisdictional claims in published maps and institutional affiliations. 